\title{
Towards Adaptive Recruitment and Engagement Mechanisms in Social Systems
}

\author{
Claudia López and Peter Brusilovsky \\ University of Pittsburgh, Pittsburgh PA 15260, USA, \\ cal95, peterb Opitt.edu, \\ WWW home page: http://adapt2.sis.pitt.edu/wiki/
}

\begin{abstract}
Although successful online communities have engaged thousands of users, designers still struggle to recruit newcomers and increase current contribution rates. Related work on encouraging contributions has drawn from Social Psychology, Sociology and Economics theories. Engagement mechanisms embed the principles of these theories, and experimental studies evaluate the impact of different mechanisms on the contribution rates. Significant differences among alternative engagement mechanisms have been found, however, the results are sometimes contradictory for different groups of users. Our hypothesis is that the effectiveness of engagement mechanisms may depend on users' characteristics, and not solely on the mechanism itself. To start exploring this hypothesis, we performed a study to evaluate the impact of recruitment and engagement messages on different users' cohorts. Levels of current participation rates and demographic data were analyzed in order to explain differences in the impact of these engagement strategies.
\end{abstract}

Keywords: online community, adaptive engagement mechanisms

\section{Introduction}

Several well-known online communities have demonstrated the potential of producing high quality products, enabling people all around the world to share content or to collaborate in geographically distributed teams. However, many other online community projects have failed in engaging enough users to achieve critical mass. Researchers have explored different ways to determine what motivates users to contribute and how to increase their levels of contribution. Previous research projects have mostly drawn from Social Psychology, Sociology and Economics $[1,13,9,5,3,18,4]$ theories. The experimental studies evaluate the impact of engagement mechanisms that embed the principles of the theories. The impact is usually measured in terms of number of new contributions added by the subjects. Significant differences in contribution rate have been found between subjects who have seen different engagement mechanisms.

A limited number of research projects have also tried to discover differences in the impact of an engagement strategy among users with different psychological characteristics. Experiments that tested the effect of displaying social information [3] showed that below-median contributors in a community increased their 
contribution at a higher rate than median and above-median contributors when exposed to social information. Furthermore, this effect was more significant for those users who present a more competitive psychological profile. On the other side, above-level contributors decreased their contribution rate. However, the most competitive above-median contributors decreased their contribution at a smaller degree. Some other studies have reported the effect of mentioning the benefits of contributing as a motivator, however the results in different studies have been contradictory. Mentioning the value of contributions increased the level of contributions in one study [13], but it decreased the contribution rate in another one [1] .

The results presented above indicate that the impact of an engagement mechanism may be dependent upon some user's characteristics as users generally have different motivations to collaborate [19]. These observations inspired us to explore adaptive engagement mechanisms in online communities. Our overall goal is to evaluate several mechanisms of adaptation such as adapting to the user's demographic data, knowledge, prior levels of contribution, and navigation patterns.

This paper reports our attempt to evaluate the effectiveness of adaptation to one aspect of user demography: a user's cultural background. Our initial hypothesis is that the effect of appealing to personal vs. community benefits may be different for users with different cultural backgrounds. For example, given the popular belief that people from Asian countries are more community-oriented, they might be more motivated to work for community goals. In contrast, people from Western countries are more concerned with personal benefits and thus could be better motivated to do work for their own benefit. This popular belief has been also supported by a multinational survey in [10]. We test this hypothesis by measuring the impact of mentioning community or personal benefits to users of different cultural backgrounds, i.e., graduate students from different home countries. Our results showed, however, that the community message was more effective in general. Moreover, the personal benefits incited more contributions from users from Asian countries. The prior level of contribution, academic program in which the user was enrolled and gender can also explain significant differences in the level of contribution after receiving the message.

The rest of the paper is organized as follows: Section 2 will describe general background about online communities, and related work on engagement strategies in online communities. Section 3 will present the study design and the system that was used as a testbed; Section 4 will detail the results of the study; Section 5 will include the discussion and future work and Section 6 will present the conclusions.

\section{Related Work}

The term online community was first defined by Rheingold in 1994 [16] as cultural aggregations that emerge when enough people bump into each other often enough in cyberspace. Since then, the Web has enabled geographically dis- 
tributed people to socially interact and create different kinds of online communities. Discussion forums, Question and Answers sites (e.g. Yahoo Answers), online social networks (e.g. Facebook and Twitter) and online community projects (e.g. Wikipedia) are good examples of successful online communities that have been able to attract thousands of active users. In addition to these well-known online communities, there are many others that were not successful $[6,2,15]$.

Several research groups have focused their efforts on finding ways to sustain online communities. Several strands of work have been studied, such as:

- how to socialize newcomers [4],

- how to encourage commitment to the community [17, 14],

- how to leverage the contribution rates $[1,11]$, and

- understanding people's motivations to engage in online communities [19].

One of the main strands of research has focused on how to encourage contributions. The main goal is to create the required amount of content (e.g. videos in Youtube or pages in Wikipedia) to provide benefits to the whole online community, including casual visitors. Simply asking for contributions is the most popular strategy. Several different ways to do this have been reported:

- broadcasting a message asking for specific contributions [1,5],

- asking specific people to do specific tasks $[1,4]$,

- emphasizing uniqueness of the user's contributions [1,12],

- providing social information and feedback $[3,13]$,

- assigning people to groups and setting group competitions $[1,7]$,

- setting personal or group goals $[1,7,18]$,

- reducing the effort required to identify tasks that are likely to be done by a user (i.e. recommend possible tasks that match the user's interests) $[9,5]$.

A more detailed description of each of these projects will be presented in the rest of this section.

In 2004, an study [1] used social psychology theories to create messages asking for more contributions to MovieLens, a movie recommender system. They run two experiments to test hypotheses borrowed from psychological theories. The first experiment tested the effect of making salient user uniqueness and mentioning the benefits of collaborating in the community. The sample was formed by users who had rated at least 3 rarely-rated movies. The lessons learned are that sending a message asking for contributions boosts the number of contributions, at least over a one-week period. Salience of uniqueness encouraged more contributions while the mention of benefit depressed ratings. The authors provided a discussion about why the mention to benefits didn't work. They argue that reminding the user about other reasons to contribute may undermine intrinsic motivations; for example, users may like to rate because it is fun, but not to help others, so mentioning that could have a negative effect. Another possible explanation is that the subjects were already committed to the community, and the message undermined their commitment by contradicting their prior beliefs regarding who benefits from each contribution. An additional feasible reason 
is that the messages were too long, thus the effort required to understand the message about benefits may have been a barrier.

The second experiment tested the effect of assigning individual and group goals. The sample was formed by active users who had logged at least once in previous 5 months. In average, these users have rated 8 movies per week. There were two conditions: group assignment and specificity of goals. Each subject may have been assigned to participate individually or as a group member. The subjects may also have been assigned to achieve an specific goal or a more ambiguous goal (e.g. "do your best"). The results showed that people who were assigned to a group contributed more than those who weren't. Additionally, setting specific goals, even the more challenging ones, generated more contributions as compared to an unspecific goal such as "do your best".

Another study of MovieLens [13] assessed the effect of displaying the value of contributions as a message in the user interface. Showing the value of contributions helped to increase the contributions. They also tested for the effect of different kinds of value: value to self, to the whole community, to a group of similar people, and to a group of different people. The message describing the value to groups was more effective than the one mentioning the value to the whole community. People also contributed more if similar people got the benefits compared to the case where dissimilar people benefited. We believe that the reason for these contradictory results might be related to users' characteristics, and not solely to the message content itself regardless the users' context.

A more recent article [3] also reported experimental studies on MovieLens. The experiments tested the effect of displaying different kinds of social information about users with different levels of participation and different competitiveness profiles. They displayed individual and community measures of contribution in the user interface. They provided two community-level measures: the median number of movie ratings in the community and the average value of Net Benefit, which is a measure of the benefits that each user has received from the community. This social feedback encouraged different levels of contribution among different user cohorts. The number of new contributions was higher in below-median users, although the contributions rate increased in all users groups. Furthermore, social information feedback changed user rating behavior. Users shifted their behavior towards the median contribution rate, but this effect was impacted by the competitive preferences of users. Within below-median users, the more competitive users increased their contribution rates to a higher degree; whereas within above-median users, more-competitive users decreased their contribution rates to a lesser degree. These research results also hint that a single engagement mechanism can have different results in different user cohorts. In fact, the authors mention the potential benefits of personalized engagement mechanisms in online communities.

Another experimental study on MovieLens aimed to compare the effect of similarity and uniqueness of users' contributions. The experiment was executed with subjects who rated in excess of 50 movies. Subjects were assigned to a group. Group formation was manipulated to generate two conditions: similar 
and dissimilar groups. Similar groups included people with similar tastes regarding movies (i.e. similar ratings for the same movies), and dissimilar groups included people with different preferences (i.e. totally different ratings for the same movies). All of the groups were asked to participate in forum discussions. The authors reported that mentioning the uniqueness of user's contributions encouraged more contributions from their part (rather than not mentioning it). Furthermore, dissimilar groups contributed more than groups that were similar. In addition, the authors reported that people who rated more movies were not necessarily the most active contributors to discussions; therefore, the willingness to contribute may also depend on the task.

MovieLens was also used as a tesbed for a task-routing algorithm [9]. A similar algorithm was later developed as a bot in Wikipedia [5]. The task-routing algorithm's goal was to reduce the amount of effort needed to participate by suggesting tasks that were needed and that might have matched with user interests. Both studies proved that task routing helped to increase the number of contributions.

In 2010, Choi et al. [4] reported the results of a study on socialization tactics in Wikiprojects. In these projects, newcomers receive welcome messages as a way to socialize them to the project and the rest of the users. The analysis compared the effect of standard messages automatically sent by the system and personalized messages sent by current contributors to the Wikiproject. The authors concluded that personalized messages from previous contributors encourage newcomers to contribute more and in a more sustainable way.

We think that a conceptual framework to describe engagement strategies can help to categorize previous research findings and to identify similarities and differences among the strategies. We propose a conceptual framework based on the characteristics of a message as shown in Table 1.

We believe that this conceptual framework and its categories can be used to guide adaptive mechanisms in the context of engagement strategies. The rest of this paper deals only with evaluating adaptation of messages to the users' characteristics. The study assesses the impact of alternative engagement strategies on different user cohorts.

\section{The Study}

This study tested the effect of sending emails with different information to users with different cultural backgrounds and different prior levels of contribution.

\subsection{The System}

We used the CourseAgent system and its users as a testbed of our study. CourseAgent [8] is a community-based study planning system for graduate students in the School of Information Sciences (iSchool) at the University of Pittsburgh. CourseAgent allows students to plan their studies and rate courses that they have taken reflecting workload and relevance to personal career goals. 
Table 1. Conceptual Framework to Characterize Related Work

\begin{tabular}{|c|c|}
\hline Category & Sub-Category \\
\hline $\begin{array}{l}\text { Sender: The person or agent that } \\
\text { sends the message. }\end{array}$ & $\begin{array}{l}\text { The system }[4,9] \\
\text { Another user }[4] \\
\text { An authority } \\
\text { A bot }[5,9]\end{array}$ \\
\hline $\begin{array}{l}\text { Receivers: The users who receive } \\
\text { the message. }\end{array}$ & $\begin{array}{l}\text { Current contributors }[3] \\
\text { Newcomers }[7,3] \\
\text { Readers, but not contributors (lurkers) }\end{array}$ \\
\hline $\begin{array}{l}\text { Style or format: The way in which } \\
\text { the content is displayed. }\end{array}$ & $\begin{array}{l}\text { Short messages vs. long messages }[1] \\
\text { Standarized vs. personalized messages }[4] \\
\text { E-mail, GUI message, pull request }[1,5]\end{array}$ \\
\hline $\begin{array}{l}\text { Information: The content that is } \\
\text { embedded in the message. }\end{array}$ & $\begin{array}{l}\text { Salience of uniqueness and dissimilarity }[1,12] \\
\text { Salience of benefits }[1,13] \\
\text { Adding social information }[13,3] \\
\text { Setting goals }[1,7,18] \\
\text { Encouraging socialization }[1,13,7] \\
\text { Reducing the cost of contributing }[9,5]\end{array}$ \\
\hline
\end{tabular}

CourseAgent serves as a communication platform and a source of knowledge about the suitability of iSchool courses to meet specific career goals.

Membership is restricted to iSchool graduate students only. A new account is created for each new student who is enrolled in one of the graduate programs at the iSchool. Recently, the system was expanded to include the graduation status. When we started the studies, there were 1256 registered users. Of those, 123 users were already graduated (according to system data), 517 user had unknown student status and 616 were current students.

Out of 1256 registered users, 175 users $(13,9 \%)$ have added at least one taken course to their study history. This is the most popular kind of contribution. By the volume of contributions, the most successful feature is adding course evaluations with respect to a specific career goal. There were 1085 contributions of this kind. These numbers show that CourseAgent is an young community that has not achieved a high number of contributions yet.

\subsection{The Study Design}

The study was designed to test the impact of recruitment and engagement messages (appealing to community benefit versus those appealing to a personal benefit) on the behavior of students with different cultural backgrounds. The sample was a subset of current iSchool graduate students. The cultural background of students was modeled by their home country (represented as a part of student demographic data). The impact was measured by monitoring the changes in the database (such as added course ratings) and tracking user actions through the system $\log$ mechanism. The latter allowed us to observe those who have not contributed, but had who logged on the system. 
The experiment manipulated the kinds of messages and the user cohorts that received each message. A user only received one message during the study, and the user's activities before and after getting the message were tracked and analyzed. All of the subjects were exposed to the same interface when log on the system after receiving the message. Therefore, the only variation in the subjects experience was the message content. Cohorts were defined by an equal distribution of users home country and the level of participation in the system before the message was sent.

The first execution of the study was run during Fall 2010, when the Spring term registration period begun. The message asked users to rate three courses they had taken before Fall 2010; therefore all users who had started their programs in Fall 2010 were removed from the subject sample. The second round of emails was sent after the end of the Fall 2010 semester (but before Spring 2011 registration was finished) to users who had started their programs in Fall 2010, so they were now able to rate courses they took during their first term. The messages that were sent in these two rounds are shown in Table 2.

Table 2. Example of Community Benefit and Personal Benefit Messages

Community Benefit Message

CourseAgent enables the students to receive recommendations from other students, as well as advice from faculty, regarding their course of study, workload, and relevance of courses. The usefulness of CourseAgent recommendations for the student community increases as users provide more information including courses they have taken, their career goals, and their ratings of courses.

We are trying to enhance the utility of CourseAgent before Spring registration starts. Please help your fellow students by adding and rating three courses you have taken and completed in the past by November 22th. Your contribution will empower the system to better recommend courses to all of the iSchool students just in time for their Spring registration.

Personal Benefit Message

CourseAgent helps you to plan your course of study wiser by keeping track of your progress towards selected career goals and by offering advice from faculty and peer students about workload and relevance of courses. The usefulness of CourseAgent increases as you provide more information about courses taken, career goals, and your ratings of courses.

We are trying to provide the best support for you before you start your Spring registration. To help us with that, please add and rate three courses you have taken and completed in the past by November $22 \mathrm{th}$. Providing three course ratings by November 22 th will help the system to present you a more complete picture of your progress (through the Career Scope tab) and better recommend you relevant courses just in time for your Spring registration. 
The study was replicated in a slightly different form with newcomers. Students whose start term was Spring 2011 received a welcome email that mentioned community benefits or personal benefits, and asked them to provide career goals and courses to be taken.

In total, e-mail messages were sent to 574 users. Six students received duplicate emails because they were students in the iSchool before, but changed to new program in Fall 2010 or Spring 2011. Although they were included twice in the subject selection of different executions of the study, they were removed from the analysis.

The students who received these messages came from 30 different home countries to pursue their graduate degrees in the iSchool. Note that in our context, the home country is not just the country of birth, but the country where students lived and studied at least until finishing their high school. Moreover, with just a few exceptions, home country is also the country where iSchool graduate students earned their undergraduate degree. As a result, in this context, student home country was used as a reasonable indication of students' cultural background. For this study, 6 groups of countries were defined considering their geographic and cultural similarities, and the number of iSchool students who came from those countries. The categories were defined as follows:

- Undefined: Students whose home country was not available at the time of the study.

- United States: Students whose home country is United States.

- Asia: Students whose home country is China (PRC), Taiwan, Republic of Korea, Japan, or Thailand.

- India: Students whose home country is India.

- Middle East: Students whose home country is Islamic Republic of Iran, Turkey, Saudi Arabia, Kuwait, or Egypt.

- Others: Students whose home country is Mexico, Libyan Arab Jamahiriya, Trinidad y Tobago, Puerto Rico, Slovakia, Singapore, Nepal, Viet Nam, Canada, Chile, Russian Federation, Poland, Ukraine, Afghanistan, Uganda, Niger, Netherlands, Bangladesh, or Yugoslavia.

\section{The Results}

As a result of the study, 32 out of 568 message receivers used the system within one week after receiving the encouragement message (0.056\%): 18 students who received the community benefits message and 14 who received the personal benefits message. Table 3 shows a detailed description of the results by country category. In our analysis of engagement, we distinguished contributions (i.e. adding a piece of information to the online community) and actions that included both contributory actions and exploratory actions such as navigation through pages. Contributions add new information to the "community wisdom" and can measure the community-benefits segment of user engagement while the total volume of actions measures overall user engagement into working with the system. Users 
can contribute by merely adding a completed or planned courses to their profiles, and by rating taken courses regarding career goals. Both tasks can be successfully done without writing English phrases. Reviews are optional in the evaluation of the courses. We did not consider the comments in the study analysis because non-native speakers may have been less confident to write a review in English, therefore it would have biased the study results.

As the table shows, overall, the community message generated more actions in the system and more contributions.

Table 3. Number of Engaged Users

\begin{tabular}{|c|c|c|c|c|c|c|c|c|c|}
\hline & & \multicolumn{2}{|c|}{ \#Messages } & \multicolumn{2}{|c|}{ \#EngagedUsers } & \multicolumn{2}{|c|}{ \#Actions } & \multicolumn{2}{|c|}{ \#Countrib. } \\
\hline & Total & Comm. & Pers. & Comm. & Pers. & Comm. & Pers. & Comm. & Pers. \\
\hline Unknown & 56 & 33 & 23 & 0 & 0 & 0 & 0 & 0 & 0 \\
\hline Asia & 66 & 27 & 39 & 2 & 4 & 91 & 78 & 42 & 50 \\
\hline India & 18 & 9 & 9 & 0 & 1 & 0 & 12 & 0 & 11 \\
\hline Middle East & 11 & 6 & 5 & 1 & 1 & 8 & 8 & 3 & 7 \\
\hline Other & 12 & 5 & 7 & 0 & 0 & 0 & 0 & 0 & 0 \\
\hline US & 405 & 205 & 200 & 15 & 8 & 234 & 119 & 108 & 54 \\
\hline Total & 568 & 285 & 283 & 18 & 14 & 333 & 217 & 153 & 122 \\
\hline
\end{tabular}

The goal of the study was to test if the community benefit message could be more effective with people from Asian countries, and the personal benefit message more effective when sent to students from Western countries. Table 4 compares the numbers related to these two specific cohorts. To our surprise, detailed data showed the opposite trend - the community benefit message engaged more users and produced more contributions among US students while the personal benefit message engaged more Asian students and produced more contributions. However, a detailed analysis of the level of actions does not produce a clear picture. Asian users who received the community message executed more actions and contributed more to the system than Asian students who received the personal message. US users provided a similar level of contributions and actions when receiving either the community benefit or the personal benefit message. A factorial logistic regression was run considering country category and kind of message as factors, and the instance of visiting the site within a week as the dependent variable. Although it seems that the community message was able to engage more US students and the personal benefit message engaged more Asian users, the predictor model using these factors didnt fit significantly better than the null model. However, the study results were still able to show significant differences in more specific cases that will be described below.

Since the number of contributions and actions are not normally distributed according to the normality tests, non-parametric tests were used to assess the 
Table 4. Ratio of Engaged Users and Level of Activity by Demographics

\begin{tabular}{|c|c|c|}
\hline Mes & US & Asia \\
\hline & \multicolumn{2}{|c|}{ \% Engaged Users } \\
\hline Community Benefit & \multicolumn{2}{|c|}{\begin{tabular}{|l|ll}
$15 / 205(0.073 \%)$ & $2 / 27(0.074 \%)$ \\
\end{tabular}} \\
\hline \multirow[t]{2}{*}{ Personal Benefit } & $8 / 200(0.04 \%)$ & $/ 39(0.103 \%)$ \\
\hline & \multicolumn{2}{|c|}{ \% Engaged New Students } \\
\hline nity Bene & $7 / 14(0.5 \%)$ & $0 / 0(0 \%)$ \\
\hline \multirow[t]{2}{*}{ Personal Benefit } & $2 / 12(0.16 \%)$ & $0 / 1(0 \%)$ \\
\hline & \multicolumn{2}{|c|}{ Mean Action Rate } \\
\hline Community Bene & $234 / 15=15.6$ & $91 / 2=45.5$ \\
\hline \multirow[t]{2}{*}{ Personal Benefit } & $119 / 8=14.875$ & $78 / 4=19.5$ \\
\hline & \multicolumn{2}{|c|}{ Mean Contribution Rate } \\
\hline Community Bene & $108 / 15=7.2$ & $42 / 2=21$ \\
\hline \multirow[t]{2}{*}{ Personal Benefit } & $54 / 8=6.75$ & $50 / 4=12.5$ \\
\hline & \multicolumn{2}{|c|}{ Mean Evaluation Rate } \\
\hline aity Bene & $31 / 15=2.07$ & $8 / 2=4$ \\
\hline Personal Benefit & $16 / 8=2$ & $26 / 4=6.5$ \\
\hline
\end{tabular}

significance of the mean differences in number of actions among different cohorts. All of the following reported results are based on non-parametric tests.

Table 5 illustrates the figures related to engaged users only. Asian students executed more actions $\left(\mathrm{M}^{1}=28.17, \mathrm{SD}^{2}=8.64\right)$ in the system than US students $(\mathrm{M}=15.35, \mathrm{SD}=2.452)$ average across the kind of messages $(\mathrm{p}<.049)$, however the difference regarding number of contribution was not significant. Furthermore, there was a significant difference among the contribution mean among the users with different levels of prior participation in the system $(\mathrm{p}<.003)$.

Table 5. Activity Levels per Engaged User

\begin{tabular}{|l|l||r|r|r|r|r|r|}
\hline Variable & Values & $\begin{array}{r}\text { Action } \\
\text { Mean }\end{array}$ & SD & Signif. & $\begin{array}{r}\text { Contrib. } \\
\text { Mean }\end{array}$ & SD & Signif. \\
\hline Home & Asia & 28.17 & 8.64 & $\mathbf{p}<\mathbf{. 0 4 9}$ & 15.33 & 6.048 & $\mathrm{p}<.145$ \\
Country & US & 15.35 & 2.452 & & 7.04 & 1.576 & \\
\hline User has & Yes & 18.33 & 4.485 & $\mathrm{p}<.113$ & 15.00 & 3.512 & $\mathbf{p}<.003$ \\
visited the & No(current students) & 20.15 & 3.840 & & 10.50 & 3.305 & \\
system before & No(new students) & 10.22 & 1.234 & & 2.22 & .969 & \\
\hline
\end{tabular}

Table 6 shows the mean number of actions and contributions executed for users with different characteristics considering the whole sample, not only the

\footnotetext{
${ }^{1}$ Median

${ }^{2}$ Standard Deviation
} 
engaged users. There was a significant difference among the mean number of actions mean among the users with different prior levels of participation in the system. The mean number of actions made by new students is much higher than the other 2 cohorts: current students who haven't visited the system and those who have visited the system before $(\mathrm{p}<.001)$. This can be explained by the information needs of new students. New students usually seek to acquire as much information as possible to make decisions, however most of them have recently arrived in the city so they do not have enough social contacts to get all the required information. The system offers them easy-to-access information about courses, and they spent most of their time looking for data in the system. However, they contribute less than current students. They do not have enough knowledge about courses to share, so their navigation pattern is more focused on browsing than contributing. Users who had contributed previously to the system contributed more $(\mathrm{M}=2.12, \mathrm{SD}=.801)$ after the message than the users who hadn't contributed before $(\mathrm{M}=.77, \mathrm{SD}=.216),(\mathrm{p}<.001)$. This can be related to the perception that the time investment for contributing is low thanks to the amount of time which they spent adding information before. They just need to update their profiles in order to get the benefits. On the other side, users who hadn't contributed before have to invest more time in the system to realize the same benefits.

Table 6. Activity Levels After Receiving a Message.

\begin{tabular}{|l|l||r|r|r||r|r|l|}
\hline Variable & Values & $\begin{array}{r}\text { Action } \\
\text { Mean }\end{array}$ & SD & Signif. & $\begin{array}{r}\text { Contrib. } \\
\text { Mean }\end{array}$ & SD & Signif. \\
\hline $\begin{array}{l}\text { User has } \\
\text { visited the }\end{array}$ & Yes & .86 & .518 & $\mathbf{p}<.001$ & .70 & .422 & $\mathrm{p}<.106$ \\
system before & No(new students) & .89 & .86 & & .46 & .142 & \\
\hline $\begin{array}{l}\text { User has } \\
\text { contributed } \\
\text { before }\end{array}$ & No & 1.80 & .589 & & .39 & .202 & \\
\hline
\end{tabular}

Regarding the students who received the community benefit message, only the previous fact of lurking (i.e. reading without adding new information to the community) or contributing to the system were factors related to statistically significant differences in the level of activity post-message. However, as the results have suggested before, the mean number of actions and contributions are higher than those computed when considering both messages. Table 7 shows these figures.

The analogous analysis for students who received the personal benefit message was executed, and the fact of contributing to the system previously is the only factor that is significant in this case. See Table 8 for a detailed description of the data. 
Table 7. Activity Levels After Receiving a Community Benefit Message

\begin{tabular}{|l|l||r|r|r||r|r|l|}
\hline Variable & Values & $\begin{array}{r}\text { Action } \\
\text { Mean }\end{array}$ & SD & Signif. & $\begin{array}{r}\text { Contrib. } \\
\text { Mean }\end{array}$ & SD & Signif. \\
\hline User has & Yes & .55 & .552 & $\mathbf{p}<.000$ & .41 & .414 & $\mathrm{p}<.658$ \\
visited the & No(current students) & 1.08 & .428 & & .57 & .240 & \\
system before & No(new students) & 2.50 & .892 & & .39 & .269 & \\
\hline User has & No & 1.06 & .360 & $\mathrm{p}<.823$ & .43 & .195 & $\mathbf{p}<.000$ \\
contributed & Yes & 1.12 & .801 & & 1.14 & .725 & \\
before & & & & & & & \\
\hline
\end{tabular}

Table 8. Activity Levels After Receiving the Personal Benefit Message

\begin{tabular}{|l|l||r|r|r||r|r|r|}
\hline Variable & Values & $\begin{array}{r}\text { Action } \\
\text { Mean }\end{array}$ & SD & Signif. & $\begin{array}{r}\text { Contrib. } \\
\text { Mean }\end{array}$ & SD & Signif. \\
\hline User has visited the & No & .77 & .216 & $\mathrm{p}<.466$ & .36 & .122 & $\mathbf{p}<.000$ \\
system before & Yes & 2.12 & .801 & & 1.22 & .453 & \\
\hline
\end{tabular}

\section{Discussion and Future Work}

Our hypothesis that community benefits message will be more effective with Asian students and the personal benefits will engage more US students was not confirmed. Unexpectedly, we found that the message tooting the community benefits engaged more US students than the personal one, however the personal benefits message engaged more Asian students. Although these differences were not significant, the pattern is surprising and we plan to continue replicating the study to verify it. We hypothesize that these unexpected trends might be also explained by our sample. Since it is not a randomized sample among people with different cultural backgrounds, it might be a biased sample. Asian students who enroll in graduate studies abroad might be more willing to work towards personal goals or it might be that they do not recognize the iSchool as their actual community. Furthermore, the US students who decide to enroll in graduate Library and Information Programs might also be a biased sample since they may be more concerned about community values than the average American.

We did find, however, one significant difference related to the demography: Asian users executed significantly more actions and added more contributions to the system than the US students regardless the kind of message they receive.

At the same time, we found a few important differences related not to user demography, but to their past experience and status in the system. Most importantly, users who had contributed to the system previously contributed significantly more after receiving the message than the users who hadn't contributed before receiving the message. We think that this is due to the fact that these users need to invest less time to improve their user profiles and enjoy the benefits of the system. On the other side, newcomers can be discouraged by the fact that they have to create their profile before getting personalized recommendations, so 
they quickly decide to stop contributing and start looking for useful information that can be obtained without a complete user profile.

Being a new student is also a significant factor in the number of actions to be executed in the system. Regarding the entire sample (not only engaged users), new students executed significantly more actions than the other cohorts. However, they did not contribute more than the others. We believe that this reflects an information-seeking behavior. As new students, they probably lack information as well as social contacts within the iSchool, so the system offers them a way to explore information that they might need. However, they do not have enough information to share yet. We see this as an opportunity. We think that engaging new students might be easier than re-engaging those that have already decided not to use the system.

Regarding the whole context of adaptive recruitment and engagement strategies, we think that this study supports the claim for an adaptive approach since it proves that different engagement strategies had different levels of effectiveness in different user cohorts. Although users' demographics seems not to be sufficient to guide the adaptation, we think that current levels of contributions are key in the selection of the user engagement mechanisms. However, there could be additional hidden personality traits or experiences which we don't measure, but which will determine higher online participation. Larger proportions of these users will inevitably join a new online community in the first place. This could explain the higher participation of current users. These variables are still personal characteristics, we just do not know what these hidden variables are yet.

Our future work is focused on implementing adaptive engagement based on contribution levels. We will test the impact of adaptive emails as well as adaptive interfaces. Furthermore, we also aim to implement adaptive mechanisms according to different users' motivations and personal traits.

\section{Conclusion}

Online community designers usually struggle to encourage users to contribute enough content to make the site sustainable. One of the most common engagement mechanisms is to send messages to current users to ask for contributions. Previous research has used the salience of benefits in the message as a motivator, however this has produced contradictory results in different studies. In this paper, we proposed that the difference could be explained by users' characteristics rather than by the message itself. We designed an experimental study to test the effectiveness of messages mentioning community benefits and personal benefits of contributing in different cohorts. The subjects were assigned to different cohorts according to their home country and level of contributions in the past. We reported on the results of the execution and replications of this study in an online community. Our original hypothesis - that community benefits message would be more effective with Asian users, and the personal benefits message more effective with US users - was not confirmed (in fact, the observed trend was the opposite). Moreover, we were not able to register reliable differences 
in actions and contributions when dividing students by demography. The only exception is the larger volume of actions performed by Asian students. However, even this observation may not be considered reliable since the overall number of engaged Asian students was low.

At the same time, we discovered that the student status in the system (new or past user) and overall level of activity (active or passive users) appear to be more reliable factors for predicting users behavior. The fact of being a newcomer to the system, having contributed before to the system or being a new student are the most significant factors in predicting the level of contribution that the messages generated.

While we are still interested in exploring the value of demographic factors in personalizing engagement strategies, we want to shift the main focus of our work to adapting the engagement strategies to the prior level of participation in the system. Another venue of research will evaluate the survival rates of the subjects of this study considering factors such as the kind of message they received and their navigation patterns. The ultimate goal is to propose an adaptive approach to increase the effectiveness of the engagement strategies.

\section{References}

1. Beenen, G., Ling, K., Wang, X., Chang, K., Frankowski, D., Resnick, P., Kraut, R.E.: Using social psychology to motivate contributions to online communities. In: Proceedings of the 2004 ACM conference on Computer supported cooperative work. pp. 212-221. CSCW '04, ACM, New York, NY, USA (2004)

2. Butler, B.: The dynamics of cyberspace: Examining and modeling online social structure, chap. When is a Group not a Group An Empirical Examination of Metaphors for Online Social Structure. Graduate School of Industrial Administration Pittsburgh: Carnegie-Mellon (1999)

3. Chen, Y., Harper, F.M., Konstan, J.A., Li, S.X.: Social comparisons and contributions to online communities: A field experiment on movielens. In: Computational Social Systems and the Internet (2007)

4. Choi, B., Alexander, K., Kraut, R.E., Levine, J.M.: Socialization tactics in wikipedia and their effects. In: Proceedings of the 2010 ACM conference on Computer supported cooperative work. pp. 107-116. CSCW '10, ACM, New York, NY, USA (2010)

5. Cosley, D., Frankowski, D., Terveen, L., Riedl, J.: Suggestbot: using intelligent task routing to help people find work in wikipedia. In: Proceedings of the 12 th international conference on Intelligent user interfaces. pp. 32-41. IUI '07, ACM, New York, NY, USA (2007)

6. Cummings, J.N., Butler, B., Kraut, R.: The quality of online social relationships. Commun. ACM 45, 103-108 (July 2002), http://doi.acm.org/10.1145/514236.514242

7. Drenner, S., Sen, S., Terveen, L.: Crafting the initial user experience to achieve community goals. In: Proceedings of the 2008 ACM conference on Recommender systems. pp. 187-194. RecSys '08, ACM, New York, NY, USA (2008)

8. Farzan, R., Brusilovsky, P.: Encouraging user participation in a course recommender system: An impact on user behavior. Comput. Hum. Behav. 27, 276-284 (January 2011), http://dx.doi.org/10.1016/j.chb.2010.08.005 
9. Harper, F.M., Frankowski, D., Drenner, S., Ren, Y., Kiesler, S., Terveen, L., Kraut, R., Riedl, J.: Talk amongst yourselves: inviting users to participate in online conversations. In: Proceedings of the 12th international conference on Intelligent user interfaces. pp. 62-71. IUI '07, ACM, New York, NY, USA (2007)

10. Hofstede, G., McCrae, R.R.: Personality and culture revisited: Linking traits and dimensions of culture. CrossCultural Research 38, 52-88 (2010)

11. Kraut, R.E., Resnick, P.: Evidence-based social design: Mining the social sciences to build online communities, chap. Encouraging Contribution to Online Communities. Cambridge, MA: MIT Press. (2011, to appear)

12. Ludford, P.J., Cosley, D., Frankowski, D., Terveen, L.: Think different: increasing online community participation using uniqueness and group dissimilarity. In: Proceedings of the SIGCHI conference on Human factors in computing systems. pp. 631-638. CHI '04, ACM, New York, NY, USA (2004)

13. Rashid, A.M., Ling, K., Tassone, R.D., Resnick, P., Kraut, R., Riedl, J.: Motivating participation by displaying the value of contribution. In: Proceedings of the SIGCHI conference on Human Factors in computing systems. pp. 955-958. CHI '06, ACM, New York, NY, USA (2006)

14. Ren, Y., Kraut, R., Kiesler, S., Resnick, P.: Evidence-based social design: Mining the social sciences to build online communities, chap. Encouraging commitment in Online Communities. Cambridge, MA: MIT Press. (2011, to appear)

15. Resnick, P., Janney, A., Buis, L.R., Richardson, C.R.: Starting an online community on demand: A case study of adding forums to a physical activity promotion program. Journal of Medical Internet Research (2010)

16. Rheingold, H.: A slice of life in my virtual community, pp. 57-80. MIT Press, Cambridge, MA, USA (1994)

17. Sassenberg, K.: Common bond and common identity groups on the internet: Attachment and normative behavior in on-topic and off-topic chats. Group Dynamics $6(1), 27-37$ (2002)

18. Wash, R., MacKie-Mason, J.: Using a minimum threshold to motivate contributions to social computing (2009)

19. Wasko, M.M., Faraj, S.: Why should i share? examining social capital and knowledge contribution in electronic networks of practice. MIS Quarterly 29 (2005) 